\title{
Infection related to intracranial pressure monitors in adults: analysis of risk factors and antibiotic prophylaxis
}

\author{
Jill A Rebuck, Kellie R Murry, Denise H Rhoney, Daniel B Michael, William M Coplin
}

Department of

Pharmacy, Detroit

Receiving Hospital and

University Health

Centre, 4201 Saint

Antoine, Detroit MI

48201, USA

J A Rebuck

K R Murry

D H Rhoney

D B Michael

W M Coplin

Department of Neurological Surgery

D B Michael

Department of

Neurology

W M Coplin

Department of Pharmacy Practice, Wayne State University College of Pharmacy and Allied Health

Professions

D H Rhoney

Wayne State University School of Medicine

D B Michael

W M Coplin

Correspondence to:

Dr D H Rhoney

d_rhoney@wayne.edu

Received 13 October 1999 and in revised form

13 April 2000

Accepted 19 April 2000
Abstract

Objective-Infection is a complication related to intracranial pressure monitoring devices. The timing, duration, and role of prophylactic antimicrobial agents against intracranial pressure monitor (ICPM) related infection have not previously been well defined. Risk factors and selection, duration, and timing of antibiotic prophylaxis in patients with ICPMs were evaluated.

Methods-Records of all consecutive patients who underwent ICPM insertion between 1993 and 1996 were reviewed. Patients included were older than 12 years with an ICPM placed for at least 24 hours. Exclusion criteria consisted of ICPM placed before admission or documented CSF infection before or at the time of insertion. Standard criteria were applied to all patients for diagnosis of CSF infection.

Results-A total of 215 patients were included, 16 (7.4\%) of whom developed CSF infection. Antibiotic prophylaxis for ICPM placement was administered to $63 \%$ of infected and $59 \%$ of non-infected patients. Vancomycin $(60 \%)$ and cefazolin (34\%) were used most often. Sixty per cent (6/16) of patients who developed infection and $45 \%$ (53/199) of those without CSF infection received their first antibiotic insertion. Risk factors for CSF infection included duration of monitoring greater than 5 days (RR 4.0 (1.3-11.9)); presence of ventriculostomy (RR 3.4 (1.0-10.7)); CSF leak (RR 6.3 (1.5-27.4)); concurrent systemic infection (RR 3.4 (1.2-9.5)); or serial ICPM (RR 4.9 (1.7-13.8)).

Conclusions-Administration of antibiotics to patients before or at the time of ICPM placement did not decrease the incidence of CSF infection. Patients found to be at greater risk for infection at our institution included duration of ICPM greater than 5 days, use of ventricular catheter, CSF leak, concurrent systemic infection, or serial ICPM.

(F Neurol Neurosurg Psychiatry 2000;69:381-384)

Keywords: antibiotic; infection; intracranial pressure; ventriculostomy

Intracranial pressure (ICP) monitoring may facilitate early and accurate intervention in neurological injury. Treatment of increased
ICP is dependent on monitoring and increases the potential for recovery. ${ }^{1}$ Devices for monitoring ICP, though, are not without complications. Infection is the primary complication encountered with ICP monitors (ICPMs); reports range from 0 to $40 \%$, and average $10 \% .^{2-16}$ Although some authors suggest antibiotic prophylaxis, studies are lacking that specifically define the timing, duration, and efficacy of prophylactic regimens. The primary objectives of this study were to (1) evaluate the observed selection, duration, and timing of various prophylactic antibiotics in patients with ICPMs and (2) to assess risk factors for CSF infection in patients with ICPMs admitted to our institution.

\section{Methods}

PATIENT SELECTION AND DEFINITION OF INFECTION

This retrospective cohort consisted of patients with ICPM placement between August 1993 and August 1996 at a university affiliated 340 bed inpatient and 92 bed emergency department level I trauma centre. This study was approved by the human investigation committee of Wayne State University. Patients were included if they were 12 years of age or older and had an ICPM in place for at least 24 hours (to exclude early deaths). Patients were excluded if they had an ICPM placed before admission, an ICPM in place for an indeterminate period of time, or a CSF infection before monitor insertion. The ICPMs used for patients in this study were placed either in the parenchyma (Camino, ${ }^{\circledR}$ NeuroCare San Diego, CA, USA) or in the ventricles as an external transducer (Medtronic, Minneapolis, MN, USA).

Definition of CSF infection was adapted from the Centers for Disease Control guidelines ${ }^{17}$ : CSF infection must have met at least one of the following two criteria: (1) presence of an organism isolated from CSF culture; and/or (2) fever $\left(>38^{\circ} \mathrm{C}\right)$ in the absence of other recognised cause with institution of appropriate antimicrobial therapy and any of the following: (a) increased white cells $(>50 \%$ polymorphonuclear leucocytes), increased protein, and/or decreased glucose $(<15 \mathrm{~g} / \mathrm{dl})$ in $\mathrm{CSF}$, or (b) organism on CSF gram stain. A concurrent infection was defined as a microorganism from an extracranial site with clinical signs/symptoms of site specific infection and demonstration of fever $\left(>38^{\circ} \mathrm{C}\right)$, peripheral leucocytosis (white blood cell count $>10$ 500/ $\mathrm{mm}^{3}$ ), and institution of appropriate antimicrobial therapy. 
Table 1 Risk factors for CSF infection: infection rate comparison of non-exposed to exposed patients

\begin{tabular}{|c|c|c|c|c|}
\hline & \multicolumn{4}{|c|}{ Risk factor present $t$} \\
\hline & No & Yes & $R R$ & $95 \%$ CI $\neq$ \\
\hline \multicolumn{5}{|l|}{ Type of initial monitor: } \\
\hline Ventriculostomy & $4 / 109(3.7)$ & $12 / 106(11.3)$ & 3.4 & $1.0-10.7^{\star}$ \\
\hline CSF leak & $13 / 205(6.3)$ & $3 / 10(30.0)$ & 6.3 & $1.5-27.4^{\star}$ \\
\hline Extracranial infection site & $7 / 151(4.6)$ & $9 / 64(14.1)$ & 3.4 & $1.2-9.5^{\star}$ \\
\hline ICP monitoring $>5$ days & $5 / 133(3.8)$ & $11 / 82(13.4)$ & 4.0 & $1.3-11.9^{\star}$ \\
\hline Patients with > 1 ICPM & $8 / 173(4.6)$ & $8 / 42(19.0)$ & 4.9 & $1.7-13.8^{\star}$ \\
\hline Skull fracture & $11 / 166(6.6)$ & $5 / 49(10.2)$ & 1.6 & $0.5-4.9$ \\
\hline Presence of multiple trauma & $9 / 95(9.5)$ & $7 / 120(5.8)$ & 0.6 & $0.2-1.7$ \\
\hline Penetrating head injury & $15 / 199(7.5)$ & $1 / 16(6.3)$ & 0.8 & $0.1-6.6$ \\
\hline $\mathrm{ICP}>20 \mathrm{~mm} \mathrm{Hg}$ & $7 / 139(5.0)$ & $9 / 76(11.8)$ & 2.5 & $0.9-7.1$ \\
\hline Antibiotic prophylaxis given & $6 / 87(6.9$ & $10 / 128(7.8)$ & 0.9 & $0.3-2.5$ \\
\hline Intracranial haemorrhage & $1 / 60(1.7)$ & $15 / 155(9.7)$ & 6.3 & $0.8-49.0$ \\
\hline \multicolumn{5}{|l|}{ Location of placement: } \\
\hline Operating room & $13 / 147(8.8)$ & $3 / 68(4.4)$ & 0.5 & $0.1-1.7$ \\
\hline Emergency department & $12 / 144(8.3 \%)$ & $4 / 71(5.6 \%)$ & 1.5 & $0.5-4.9$ \\
\hline Intensive care unit & $7 / 139(5.0 \%)$ & $9 / 76(11.8 \%)$ & 0.4 & $0.1-1.1$ \\
\hline
\end{tabular}

${ }^{\star} \mathrm{p}<0.05$.

tRepresented as the infection rate (\%) in those without a particular characteristic and in those with the above characteristic.

$\ddagger$ Univariate logistic regression. All values $\mathrm{p}>0.05$ for multivariate logistic regression.

CLINICAL DATA AND RISK FACTORS

Data were collected for each patient on standardised forms and included age, sex, primary neurological diagnosis, baseline Glasgow coma scale (GCS) score, patient disposition at discharge, and Glasgow outcome scale (GOS) score. Factors suspected as risks for CSF infection from previous studies were evaluated. ${ }^{2-4}$ 6-16 These putative risk factors included hospital location of ICPM placement (for example, emergency department, operating room, or ICU), type of ICPM (intraventricular or intraparenchymal), number of serial ICPMs/ patient, duration of ICPM, CSF infection, skull fracture, trauma, penetrating head injury, intracranial haemorrhage, CSF leak, sustained ICP greater than $20 \mathrm{~mm} \mathrm{Hg}$, and neurosurgical/non-neurosurgical operative procedures. Presence of positive blood, urine, sputum, sinus, catheter tip, or wound cultures were also recorded along with the organism(s) cultured from these sites to assess their possible relation to CSF infection.

\section{ANTIBIOTIC PROPHYLAXIS}

Timing of antibiotic prophylaxis was evaluated using documentation times recorded for administration of the antibiotic in relation to the time of ICPM placement. Further assessment included other antibiotics administered during the duration of monitor placement unrelated to monitor related prophylaxis. The type of antibiotic, dosage, frequency, duration, total number of doses, and time administered in relation to monitor placement were recorded for all antibiotics.

STATISTICAL ANALYSIS

Data were entered, validated, and analyzed using SPSS ${ }^{\circledast}$ software (version 7.5 SPSS Inc., Chicago, IL, USA). The significance of observed differences between the infection and no-infection patient groups was analyzed using $\chi^{2}$ and two tailed Fisher's exact tests. Univariate and multiple logistic regression analysis were performed to determine factors relevant to risk of CSF infection, represented by relative risks (RRs) and 95\% confidence intervals
(95\% CIs). Ordinal variables were analyzed using the Mann-Whitney $U$ test. The variables analyzed in relation to antibiotic prophylaxis were timing, duration of administration, and type of antibiotic chosen. A p value $<0.05$ was considered statistically significant.

\section{Results}

PATIENT CHARACTERISTICS

A total of 258 medical charts were reviewed. Forty three patients were excluded because of: ICPM in place for less than 24 hours (31); positive CSF culture on admission (five); ICPM placed at another hospital before admission (4); and indeterminate duration of ICPM placement (three). The eligible study population consisted of 215 patients who underwent insertion of 272 ICPMs. Sixteen patients $(7.4 \%)$ developed a CSF infection when the ICPM was present. Patient characteristics were similar between the infected and non-infected groups (mean age: 43 (SD 17) $v$ 44 (SD 15) years; sex: $68 v 69 \%$ male; baseline median GCS: 7 (range 3-15) v 6.5 (range 2-15); and median GOS: 3 (range 1-5) v 3 (range 1-4)). Primary neurological diagnoses were similar $(\mathrm{p}>0.05)$ and included intracranial haemorrhage (95), traumatic brain injury (58), aneurismal subarachnoid haemorrhage (25), gunshot wound (13), tumour (11), hydrocephalus (four), global cerebral ischaemia not otherwise specified (four), cerebellar infarct (four), and abscess (one).

EVALUATION OF PUTATIVE RISK FACTORS

Potential independent risk factors for ICPM related infection were evaluated (table 1). The type of ICPM used was related to developing CSF infection. Rate of infection was higher $(11.3 v 3.7 \%)$ for those patients who underwent initial ICPM placement with a ventriculostomy (RR 3.4 (95\% CI 1.0-10.7)). The presence of a CSF leak also resulted in a strong association with infection (RR 6.3 (95\% CI 1.5-27.4)). Additionally, patients with concurrent non-CNS sites of infection, specifically pneumonia and urinary tract infections, were more likely to develop ICPM related infections (RR 3.4 (95\% CI 1.2-9.5)). The organisms cultured were those expected at the specific site of infection, and were distinct from those organisms cultured from the CSF in $78 \%$ of patients. The incidence of infection was increased in patients requiring monitoring for greater than 5 days (RR $4.0 \quad(95 \%$ CI 1.3-11.9)). Patients with more than one ICPM were also found to be at an increased risk for infection (RR 4.9 (95\% CI 1.7-13.8)).

MICROBIOLOGY

Bacteria isolated from patients who developed CSF infection included five cases of Staphylococcus epidermidis ( $80 \%$ methicillin resistant), two cases of Acinetobacter baumannii, and one case each of Corynebacterium jeikeium, Enterobacter cloacae, Klebsiella pneumoniae, Proteus mirabilis, and Stenotrophomonas maltophilia. Four patients without an organism isolated met inclusion for having CSF infection as previously defined. 


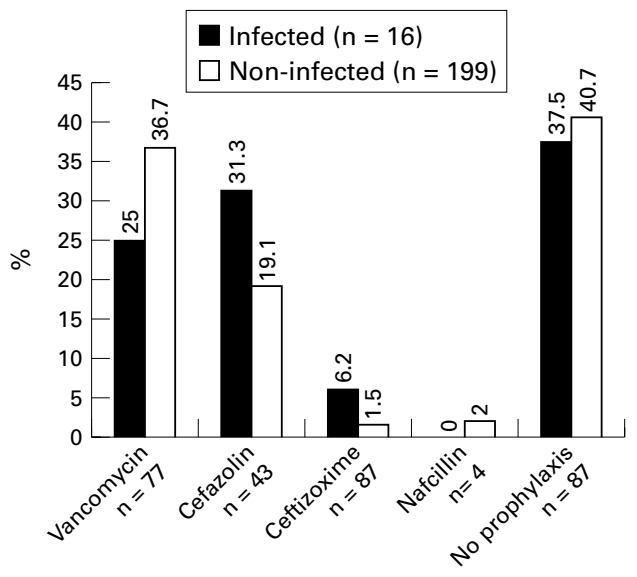

Comparison of percentage of antibiotic prophylaxis in all patients in relation to CSF infection.

Table 2 Characteristics of antibiotic prophylaxis

\begin{tabular}{|c|c|c|}
\hline Characteristic $^{\star}(n(\%))$ & $\begin{array}{l}\text { Non-infected } \\
(n=118)\end{array}$ & $\begin{array}{l}\text { Infected } \\
(n=10)\end{array}$ \\
\hline \multicolumn{3}{|l|}{ Timing of antibiotic prophylaxis: } \\
\hline$>2$ hours before ICPM insertion & $7(5.9)$ & $0(0)$ \\
\hline $0-2$ hours before ICPM insertion & $53(44.9)$ & $6(60.0)$ \\
\hline First dose given after ICPM insertion & $58(49.2)$ & $4(40.0)$ \\
\hline \multicolumn{3}{|l|}{ Duration of antibiotic prophylaxis: } \\
\hline One dose before ICPM insertion & $30(25.4)$ & $4(40.0)$ \\
\hline Periprocedural administration & $23(19.5)$ & $3(30.0)$ \\
\hline Entire duration of ICPM placement & $41(34.8)$ & $2(20.0)$ \\
\hline Continued after ICPM discontinued & $24(20.3)$ & $1(10.0)$ \\
\hline
\end{tabular}

ANTIBIOTIC PROPHYLAXIS

A total of $128 / 215(59.5 \%)$ patients received antibiotics around the first ICPM placement. In the group with CSF infection, 10 of 16 patients $(62.5 \%)$ received antibiotic prophylaxis compared with 118 of 199 patients $(59.3 \%)$ in the non-infected group $(p>0.05)$. The choice of antimicrobial agent(s) for prophylaxis is shown in the figure.

Timing of antibiotic prophylaxis was similar between both groups (table 2). Duration of antibiotic prophylaxis (number of doses) as related to ICPM placement was also compared in table 2. Most patients who developed an ICPM related infection received either one dose $(40 \%)$ or a specific number of periprocedural antibiotic prophylactic doses $(30 \%)$.

\section{Discussion}

The results of our investigation indicate that prophylactic administration of antibiotics around ICPM insertion may not be useful in preventing CSF infection. Similar percentages of patients in both groups received their first antimicrobial dose within 2 hours before ICPM insertion. We also found type of ICPM (intraventricular $v$ intraparenchymal), CSF leak, concurrent infection, greater than 5 day duration, and serial ICPM placement were all independent risk factors for development of CSF infection.

Antibiotic administration within 2 hours before ICPM placement is necessary to allow for adequate tissue concentrations. ${ }^{18}$ Whereas prophylaxis was intended to be administered within this period, $40 \%$ of patients developing infections and $49.2 \% \quad(p>0.05)$ of those non-infected patients received their first antibiotic dose after ICPM placement, potentially affecting our results. The difference in time between prescribing an antimicrobial and administration of the agent in relation to ICPM insertion must be considered.

The ideal duration of antibiotic prophylaxis in patients with ICPMs has yet to be identified. Poon et al reported a decreased incidence of infection in patients randomised to prophylaxis for the duration of ICPM placement compared with perioperative antimicrobial dosing. ${ }^{19}$ In our study, the largest percentage $(56 \%)$ of patients receiving antibiotics for the duration of ICPM placement or beyond were in the non-infected group. Of those infected, most $(70 \%)$ received either one dose or a short course of a specific number of antibiotic doses. Interestingly, $30 \%$ of those patients who developed CSF infection received prophylaxis for at least the entire duration of ICPM placement compared with $55.1 \%$ of those who did not develop CSF infection. Duration of prophylaxis had no significant influence on development of infection.

The effect of antibiotic prophylaxis on rate of ICPM related infections has only been discussed in small patient populations. ${ }^{2}$ 6 9-11 13-16 $^{13}$ Studies with similar numbers of infected versus non-infected patients receiving prophylaxis found that the use of antibiotics did not influence outcome. ${ }^{2614}$ We also did not find a significant relation between whether or not patients received antibiotic prophylaxis and rate of ICPM related infection. Based on our microbiology results, prophylaxis with vancomycin and an agent with appropriate gram negative coverage would be required. However, broad spectrum antibiotics may favour growth of nosocomial pathogens and increase antibiotic resistance potential. ${ }^{19} 20$

The most often evaluated risk factor for ICPM related infection is the duration of monitoring. Several authors have related length of time ICPMs were left in place to infection. ${ }^{2} 68911121416$ Mayhall et al found a higher risk of infection in patients with ventricular catheters in place greater than 5 days. ${ }^{10}$ We also found that after 5 days or more of monitoring, an increased risk for infection occurred in our patient population; however, we did not evaluate the effect of catheter exchange on infection rate. Furthermore, we excluded all patients with ICPMs in place for less than 24 hours, which may have affected our results as these patients likely represented the subset with the highest mortality rates. Additionally, we found the type of ICPM used had a significant relation to the development of infection. Although ventricular catheters have a higher incidence of infection due to deep brain penetration, they are accurate and reliable measures of ICP and allow ventricular access for CSF drainage. ${ }^{21}$

This study was performed retrospectively using information obtained from medical records. A prospective study would have allowed for more detailed and controlled data collection. Evaluation of CSF infection was potentially confounded by the difficulties 
inherent in retrospectively distinguishing between true infection and inflammation. The study was conducted in a level I trauma centre with a highly complex neurosurgical department; it was assumed that all personnel had adequate ICPM training and experience. Another limitation was the inability to assess influence of tunnelling the catheter tubing on decreasing rate of infection. Furthermore, the small sample size of the infected patient group limited detection of true statistical significance. An extensive prospective randomised trial is needed to assess the full impact of antibiotic prophylaxis.

\section{Conclusion}

The patients at greater risk for monitor related infection within our institution included those with the following features: duration of monitoring greater than 5 days; presence of ventriculostomy; CSF leak; concurrent infection; or serial ICPM placement. At our institution, administration of antibiotics to patients before or at the time of ICPM placement did not decrease the incidence of CSF infection. To conclusively demonstrate whether administration of antibiotic prophylaxis in ICPM placement significantly affects the risk of CSF infection, a randomised prospective multicentre study is necessary.

We acknowledge the assistance of Fran Patton, and the late Catherine Dowling, in keeping records of all patients with ICPM devices placed at our institution.

1 Bullock R, Chestnut RM, Clifton G, et al. Guidelines for the management of severe head injury. If Neurotrauma 1996;13:667-92

2 Aucoin PJ, Kotilainen HR, Gantz NM, et al. Intracranial pressure monitors: epidemiologic study of risk factors and infections. Am F Med 1986;80:369-76.
3 Bader MK, Littlejohns L, Palmer S. Ventriculostomy and intracranial pressure monitoring: in search of a $0 \%$ infection rate. Heart Lung 1995;24:166-72.

4 Bogdahn V, Lau W, Hassel W, et al. A: Continuous pressure controlled external ventriculostomy drainage for treatment of acute hydrocephalus: evaluation of risk factors. Neurosurgery 1992;31:898-904.

5 Chan KH, Mann KS. Prolonged therapeutic external ventricular drainage: a prospective study. Neurosurgery 1988;23:436-8.

6 Clark WC, Muhlbauer MS, Lowrey R, et al. Complications of intracranial pressure monitoring in trauma patients. Neurosurgery 1989;25:20-4.

7 Franges EZ, Beideman ME. Infections related to intracranial pressure monitoring. $\mathcal{F}$ Neurosci Nurs 1988;20:94-103.

8 Holloway KL, Barnes T, Choi S, et al. Ventriculostomy infections: the effect of monitoring duration and catheter exchange in 584 patients. F Neurosurg 1996;85:419-24.

9 Kanter RK, Weiner LB, Patt AM, et al. Infectious complications and duration of ICP monitoring. Crit Care Med 1985; 13:837-9.

10 Mayhall GC, Archer NH, Lamb VA, et al. Ventriculostomy related infections: a prospective epidemiologic study. $N$ Engl f Med 1984;310:553-9.

11 Rosner M, Becker DP. ICP monitoring: complications and associated factors. Clin Neurosurg 1976;23:494-519.

12 Schultz M, Moore K, Foote AW. Bacterial ventriculitis and duration of ventriculostomy catheter insertion. 7 Neurosci Nurs 1993;25:158-64.

13 Smith RW, Alksne JF. Infections complicating the use of external ventriculostomy. I Neurosurg 1976;44:567-70.

14 Stenager E, Gerner-Schmidt P, Kock-Jensen C. Ventriculostomy-related infection: an epidemiological study. Acta Neurochir (Wien) 1986;83:20-3.

15 Winfield J, Rosenthal P, Kanter R, et al. Duration of intracranial pressure monitoring does not predict daily risk of infectious complications. Neurosurgery 1993;33:424-31.

16 Wyler AR, Kelly WA. Use of antibiotics with external ventriculostomies. $\mathcal{F}$ Neurosurg 1972;37:185-7.

17 Garner JS, Jarvis WR, Emori TG, et al. CDC definitions for nosocomial infections. Am f Infect Control 1988;16:128-40.

18 Classen DC, Evans RS, Pestotnik SL, et al. The timing and prophylactic administration of antibiotics and the risk of surgical-wound infection. N Engl f Med 1992;326: 281-6.

19 Poon WS, Ng S, Wai S. CSF antibiotic prophylaxis for neurosurgical patients with ventriculostomy: a randomized study. Acta Neurochir Suppl (Wien) 1998;71:146-8.

20 Ambrose PG, Owens RC, Quintiliani R, et al. Antibiotic use in the critical care unit. Crit Care Clin 1998;14:283-308.

21 Lyons MK, Meyer FB. Cerebrospinal fluid physiology and the management of increased intracranial pressure. Mayo Clin Proc 1990;65:684-707. 